\title{
The effects of external disturbances on the performance and chaotic behaviour of industrial FCC units
}

\begin{abstract}
The dynamic behaviour of an industrial Type IV fluid catalytic cracking for the production of gasoline unit is investigated for a case where the air feed temperature is periodically forced. The investigation concentrates on the behaviour of the system for a case of bistability for the autonomous system with special emphasis on the effect of forcing on the periodic attractor of the autonomous system. When the centre of forcing is very close to the homoclinical termination point of the autonomous periodic attractor, period-doubling mechanism and Type 1 intermittency have been identified as the routes to chaos for this six-dimensional (6D) system. Chaotic behaviour occurs at very low forcing amplitudes which simulate small disturbances that are unavoidable in the operation of any industrial unit. While in certain ranges of the values of the forcing amplitudes the output amplitudes of the forced system are higher than their counterparts in the autonomous system, other regions show the opposite behaviour. Average gasoline yield in the bistability region for the attractor resulting from the forcing of the autonomous periodic attractor is much higher than that resulting from forcing the autonomous static attractor. This yield is very close to that obtained with the optimum steady state which is unstable and requires prohibitively high values of controller gains to be stabilized.
\end{abstract}

Keyword: External disturbances; Air feed temperatures; Fluid catalytic cracking; Periodic forcing; Chaotic behaviour 